\title{
Methylene blue inhibits nucleation and elongation of SOD1 amyloid fibrils
}

\author{
Greta Musteikyte $^{1,2}$, Mantas Ziaunys ${ }^{2}$, Vytautas Smirnovas ${ }^{\text {Corresp. } 2}$ \\ 1 Department of Chemistry, University of Cambridge, Cambridge, United Kingdom \\ 2 Life Sciences Center, Institute of Biotechnology, Vilnius University, Vilnius, Lithuania \\ Corresponding Author: Vytautas Smirnovas \\ Email address: vytautas@smirnovas.info
}

Protein aggregation into highly-structured amyloid fibrils is linked to several neurodegenerative diseases. Such fibril formation by superoxide dismutase I (SOD1) is considered to be related to amyotrophic lateral sclerosis, a late-onset and fatal disorder. Despite much effort and the discovery of numerous anti-amyloid compounds, no effective cure or treatment is currently available. Methylene blue (MB), a phenothiazine dye, has been shown to modulate the aggregation of multiple amyloidogenic proteins. In this work we show its ability to inhibit both the spontaneous amyloid aggregation of SOD1 as well as elongation of preformed fibrils. 
1 Methylene Blue Inhibits Nucleation and Elongation of SOD1

2 Amyloid Fibrils

4 Greta Musteikyte $^{1,2}$, Mantas Ziaunys ${ }^{1}$ and Vytautas Smirnovas ${ }^{1 *}$

$6{ }^{1}$ Institute of Biotechnology, Life Sciences Center, Vilnius University, Saulètekio av. 7, Vilnius, 7 LT-10257

8 2Department of Chemistry, University of Cambridge, Cambridge CB2 1EW, UK

Corresponding Author:

11 Vytautas Smirnovas

12 Sauletekio al. 7, Vilnius, LT-10257, Lithuania

Email address: vytautas.smirnovas@bti.vu.1t

\section{Abstract}

Protein aggregation into highly-structured amyloid fibrils is linked to several neurodegenerative diseases. Such fibril formation by superoxide dismutase I (SOD1) is considered to be related to amyotrophic lateral sclerosis, a late-onset and fatal disorder. Despite much effort and the discovery of numerous anti-amyloid compounds, no effective cure or treatment is currently available. Methylene blue (MB), a phenothiazine dye, has been shown to modulate the aggregation of multiple amyloidogenic proteins. In this work we show its ability to inhibit both the spontaneous amyloid aggregation of SOD1 as well as elongation of preformed fibrils.

\section{Introduction}

Lou Gehrig's disease or ALS (amyotrophic lateral sclerosis) is a late-onset fatal neurodegenerative disorder that results in upper motor neuron death and leads to gradual paralysis of the whole body. ALS is diagnosed for patients averagely at the age of 55, lasts for 35 years and eventually leads to death (Taylor, Brown \& Cleveland, 2016). ALS occurs in both familial and sporadic form. The inherited form (fALS) accounts for 5-10\% cases and is associated with mutations in more than 50 genes (Taylor, Brown \& Cleveland, 2016; Smith et al., 2017), while the sporadic form (sALS) is diagnosed in all other cases (Peters \& Brown Jr, 2015). The incidence of the disease is 1.9 cases of 100000 per year and it is estimated that in 2040 the number of individuals suffering from the disorder will reach 376674 (Arthur et al., 2016). Currently, there are no effective therapies for ALS; with only Riluzole and Edaravone being FDA approved drugs. However, they only prolong patient survival without curing the 
disease completely (Miller et al., 2002; Rothstein, 2017; Fang et al., 2018; Dash, Babu \& Srinivas, 2018; Jaiswal, 2019).

SOD1 (superoxide dismutase I) is a $32-\mathrm{kDa}$ homodimer which plays an antioxidative role in the cell. SOD1 was the first gene to be associated with ALS in 1993 (Rosen et al., 1993) and more than 180 disease-related mutations have been reported so far (Pansarasa et al., 2018). It is still considered as one of the most commonly associated genes with the disorder (Renton, Chiò \& Traynor, 2014). A significant number of studies revealed ALS association with SOD1 misfolding and aggregation (Rakhit \& Chakrabartty, 2006; Rotunno \& Bosco, 2013; Lee \& Kim, 2015; Pickles et al., 2016; Pansarasa et al., 2018; Paré et al., 2018). According to the gain-of-function hypothesis, misfolded SOD1 forms cross- $\beta$-sheet rich amyloid deposits in the central nervous system (Peters \& Brown Jr, 2015). Such deposits impede clearance of neurotransmitter glutamate after nerve signal transduction (Boillée, Vande Velde \& Cleveland, 2006; Zarei et al., 2015), inhibit oxidative phosphorylation (Mattiazzi et al., 2002), disrupt axonal mitochondrial transport (Maniecka \& Polymenidou, 2015) and eventually lead to motor neuron death.

Different strategies are employed in drug discovery in the field of neurosciences. One of them is direct targeting of a disease-associated protein. This is realized by looking for agents that stabilize the native structure of the protein (Maurer et al., 2018) or screening for aggregation inhibitors and fibrillization pathway modulators. In the context of ALS, SOD1 is considered to be an extremely important therapeutic target to treat this particular disease (Liu et al., 2012; Pansarasa et al., 2018). A number of studies demonstrate SOD1 protein stabilizing and/or aggregation hampering effects caused by both synthetic and natural compounds. These include curcumin (Bhatia et al., 2015), flavonoids (Zhuang et al., 2016), epigallocatechin-3-gallate (Srinivasan \& Rajasekaran, 2017), cisplatin (Banci et al., 2012), azauracil and uracil derivatives (Nowak et al., 2010), isoproterenol, 5-fluorouridine (Wright et al., 2013) and libraries of other compounds (Ray et al., 2005; Anzai et al., 2016).

Emerging potential of a small-molecule compound methylene blue (MB) as a therapeutic agent in the field of neurodegenerative disorders has also been discovered recently. MB is a phenothiazine dye used in medicine for various purposes ranging from nervous tissue visualization during surgery to methemoglobinemia, ifosfamide-induced toxicity, malaria and vasoplegic syndrome treatment (Oz et al., 2011). Its pharmacokinetic properties are well known; the typical oral dose in clinics ranges between 50-300 $\mathrm{mg}$. The drug crosses the blood-brain barrier and is reported to interact with nitric oxide synthase, butyrylcholinesterase, acetylcholinesterase; it is also an inhibitor of voltage-gated calcium channels, adrenergic, NMDA, histamine $\mathrm{H} 1 / \mathrm{H} 2$ and serotonin receptors (Yamashita et al., 2009; Oz, Lorke \& Petroianu, 2009; Oz et al., 2011).

A range of studies report an inhibitory effect of $\mathrm{MB}$ on aggregation of various neurodegenerative disease-related proteins. It has been shown that MB reduces the amount of TDP-43 aggregates in the SH-SY5Y cell line (Yamashita et al., 2009) and recovers motor neuron function in Danio 
rerio and Caenorhabditis elegans ALS disease models (Vaccaro et al., 2012, 2013). It has also been demonstrated that the compound inhibits huntingtin aggregation in vitro, increases the survival rate of mutant huntingtin transduced primary neuron culture and shows a positive effect in Huntington's disease models in vivo (Sontag et al., 2012). MB also inhibits Alzheimer's and Parkinson's disease-related tau aggregation and plaque formation (Wischik et al., 1996; Akoury et al., 2013; Crowe et al., 2013). The compound has been shown to impede Alzheimer's diseaseassociated amyloid $\beta(\mathrm{A} \beta)$ oligomerization as well. MB modulates the $A \beta$ aggregation pathway by facilitating fibrillization of the peptide, therefore reducing the formation of highly toxic oligomer species (Necula et al., 2007). The effect of MB was also demonstrated on both the native structure, as well as aggregation of a model amyloidogenic protein - lysozyme (Saha et al., 2018). Three studies report on the effect of MB on SOD1 G93A mice (Lougheed \& Turnbull, 2011; Audet, Soucy \& Julien, 2012; Dibaj et al., 2012). However, this particular mutation accounts for only a minor proportion of fALS cases (Renton, Chiò \& Traynor, 2014). To our knowledge, there are no studies reporting about MB effect on wild-type SOD1 (wtSOD1) aggregation. This subject is of particular importance since most ALS cases (90-95\%) are associated with wild-type proteins (Renton, Chiò \& Traynor, 2014; Peters \& Brown Jr, 2015).

In this study, we demonstrate for the first time that MB facilitates wtSOD1 "off-pathway" aggregation in vitro under destabilizing, amyloid-formation favouring conditions and give insights into the underlying molecular mechanism of this particular phenomenon.

\section{Materials and Methods}

\section{Stock solution preparation}

Methylene blue (Sigma-Aldrich) was dissolved in deionized water in an approximate concentration of $10 \mathrm{mM}$ and stored in the dark. The exact concentration was measured using a diluted MB solution with a UV-1800 Shimadzu spectrophotometer in a $1 \mathrm{~cm}$ pathlength cuvette $\left(\varepsilon_{664}=74028 \mathrm{M}^{-1} \cdot \mathrm{cm}^{-1}\right)$. The stock solution was filtered through a $0.2 \mu \mathrm{m}$ syringe filter (Kinesis) before use.

\section{SOD1 synthesis and purification}

Recombinant C-terminally his-tagged SOD1, cloned in pET303 vector, was expressed in E. coli BL21 Star (DE3) strain (Invitrogen). Culture from stock was grown overnight in ZYM-5052 autoinduction media (Studier, 2005) at $+37^{\circ} \mathrm{C}$. Cells were harvested by centrifugation for $30 \mathrm{~min}$ at $6000 \mathrm{rpm}$ (HeroLab), $4^{\circ} \mathrm{C}$. The culture was first homogenized mechanically and then sonicated (VS70/T probe, Bandelin) in $50 \mathrm{mM}$ sodium phosphate, $100 \mathrm{mM} \mathrm{NaCl} \mathrm{pH} 7.5$ buffer. Cell debris was pelleted by centrifugation for $30 \mathrm{~min}$ at $18000 \mathrm{rpm}, 4^{\circ} \mathrm{C}$. The supernatant was mixed with pre-equilibrated, $\mathrm{Ni}^{2+}$ ion loaded IMAC resin (GE Healthcare) and left at $4{ }^{\circ} \mathrm{C}$ to equilibrate. The resin was loaded into GE Healthcare HiScale 26/40 column, the column was connected to ÄKTApurifier chromatographic system and target protein eluted with $200 \mathrm{mM}$ imidazole step gradient. ApoSOD1 was obtained as described (Chattopadhyay et al., 2008) with 
111 slight modifications. Briefly, SOD1 was dialysed in $100 \mathrm{mM}$ acetate, $50 \mathrm{mM} \mathrm{NaCl}, 10 \mathrm{mM}$

112 EDTA pH 3.8 buffer overnight; then in $100 \mathrm{mM}$ acetate, $50 \mathrm{mM} \mathrm{NaCl}, 50 \mathrm{mM}$ EDTA pH 3.8

113 buffer for 4 hours and two times in $10 \mathrm{mM}$ potassium phosphate $\mathrm{pH} 7.4$ buffer. Dialysed protein

114 was filtered through a $0.22 \mu \mathrm{m}$ filter (Millipore) and stored at $-80^{\circ} \mathrm{C}$.

115

116

117

118

119

120

121

122

123

124

125

126

127

128

129

130

131

132

133

134

135

136

137

138

139

140

141

142

143

144

145

146

147

\section{Development of SOD1 aggregation assay}

Wild-type metallated and disulfide-oxidized superoxide dismutase I is known to be an extremely stable enzyme with a melting temperature of $92^{\circ} \mathrm{C}$ (Culik et al., 2018) and retains its activity in high concentrations of denaturing agents (Arnesano et al., 2004). Therefore, it is difficult to follow aggregation of wtSOD1 in vitro under physiological conditions in real-time. What is more, fibrillization kinetics of SOD1 are difficult to reproduce due to competing aggregation pathways and process stochasticity (Abdolvahabi et al., 2016). In this study, we have developed an assay allowing one to follow rapid and reproducible SOD1 kinetics at physiological pH (see Methods section). One key component used in the assay is a disulfide reducing agent, since disulfide reduction in SOD1 leads to destabilization of the SOD1 dimer and facilitates fibrillization (Furukawa, Torres \& O'Halloran, 2004; Sheng et al., 2013; Culik et al., 2018). Another key component is guanidine hydrochloride $(\mathrm{GuHCl})$, a chaotropic agent that accelerates SOD1 aggregation (Sheng et al., 2013) and in our case, strongly promotes SOD1 fibrillization and prevents "off-pathway" oligomer formation (Fig. S1). In the absence of GuHCl, SOD1 forms round-shaped oligomers (Fig. S1A), whereas in the presence of $0.5 \mathrm{M} \mathrm{GuHCl}$, thread-like amyloid fibers are formed (Fig. S1B). This most likely occurs due to chaotropic agent facilitated SOD1 unfolding and obtainment of a conformation that is prone to aggregation into intermolecular $\beta$-sheet rich amyloid fibers. In other words, $\mathrm{GuHCl}$ promotes the fibrillization pathway, but not the "off-pathway" oligomer formation. In our study, demetallated and disulfide bond reduced SOD1 is used, since demetallation, as well as disulfide bond reduction, leads to protein destabilization and an increased propensity to aggregate (Furukawa \& O'Halloran, 2005; Sheng et al., 2013). To confirm that purified SOD1 is in the apo form, a thermal unfolding assay was performed (see Supplementary Methods section). The measured melting temperature of SOD1 is $50.3 \pm 0.1^{\circ} \mathrm{C}$ and corresponds to a disulfide-oxidized and demetallated form of the enzyme; this is in agreement with the data presented in literature (Fig. S2, (Furukawa \& O'Halloran, 2005)). These SOD1 destabilizing conditions do not reflect the biological environment that the protein is found in vivo (apart from the $\mathrm{pH}$ value), however, theyare necessary to obtain aggregation kinetic data and examine the effect of $\mathrm{MB}$ in a reasonable timescale and with minimal stochasticity.

\section{SOD1 aggregate preparation}

wtSOD1 aggregates were prepared by diluting $200 \mu \mathrm{M}$ protein in $10 \mathrm{mM}$ potassium phosphate buffer containing $0.5 \mathrm{M} \mathrm{GuHCl}$ and $5 \mathrm{mM}$ DTT. Solutions were incubated at $60^{\circ} \mathrm{C}$ under shaking conditions at $600 \mathrm{rpm}$ (Ditabis MHR 23 shaker, Fisher Scientific) for 72 hours. Mixtures 
148

149

150

151

152

153

154

155

156

157

158

159

160

161

162

163

164

165

166

167

168

169

170

171

172

173

174

175

176

177

178

179

180

181

182

183

containing MB and/or preformed seed were prepared by adding $400 \mu \mathrm{M}$ to the buffer and/or $5 \%$ pre-formed SOD1 fibrils respectively.

\section{ThT fluorescence assays}

Aggregation experiments were carried out with $200 \mu \mathrm{M}$ apoSOD1 monomer in $10 \mathrm{mM}$ potassium phosphate, $0.5 \mathrm{M} \mathrm{GuHCl}, 5 \mathrm{mM}$ DTT buffer, $50 \mu \mathrm{M}$ of Thioflavin $\mathrm{T}$ and $0-400 \mu \mathrm{M}$ of MB. For seeded aggregation experiments, $5 \%$ of ultrasonicated fibrils were added into reaction mixtures and mixed thoroughly. Aliquots of $100 \mu \mathrm{l}$ from aggregation mixtures were poured into a 96-well low-binding microplate (Corning 3881). ThT fluorescence (excitation at $442 \mathrm{~nm}$, emission at $482 \mathrm{~nm}$ ) was monitored at $60^{\circ} \mathrm{C}$ in continuous shaking conditions every 5 or 10 minutes in Biotek Synergy H4 Multi-Mode microplate reader. Seeded aggregation experiments were carried out at $60^{\circ} \mathrm{C}$ under quiescent conditions in RT-PCR analyzer (Rotor Gene Q).

Dye binding assays

Before any dye-binding assay, $2 \mathrm{ml}$ of SOD1 aggregates were dialyzed 4 times in $1 \mathrm{~L}$ of $10 \mathrm{mM}$ potassium phosphate buffer, $\mathrm{pH} 7.4$ at room temperature. In Congo Red binding assay, $10 \mathrm{ul}$ of aggregates are mixed with $1 \mathrm{ml} 10 \mathrm{mM}$ potassium phosphate buffer containing $10 \mu \mathrm{M}$ Congo Red. The sample is incubated for $15 \mathrm{~min}$ at room temperature, then light absorption in the 400$700 \mathrm{~nm}$ range is measured (Shimadzu UV-1800 spectrophotometer). Nile Red binding assay is performed the same way, except that $10 \mu \mathrm{M}$ of Nile Red is added to the buffer instead of Congo Red. The dye is excited with $530 \mathrm{~nm}$ light and fluorescence emission is measured at 550-800 nm range. In ThT assay, $50 \mu \mathrm{M}$ of ThT is added instead of Congo Red; fluorescence emission is detected in 450-600 $\mathrm{nm}$ range after excitation with $440 \mathrm{~nm}$ light beam.

\section{Atomic force microscopy}

$30 \mu \mathrm{l}$ of the sample was deposited on freshly cleaved mica and left to adsorb for $5 \mathrm{~min}$; followed by rinsing with distilled water and gentle drying using airflow. AFM images were recorded in the Tapping-in-Air mode at a drive frequency of $300 \mathrm{kHz}$, with the Dimension Icon microscope (Bruker).

\section{Fourier transform infrared spectroscopy}

A buffer of prepared fibers was exchanged into $\mathrm{D}_{2} \mathrm{O}$ by a series of centrifugation and rinsing steps. Shortly, fibrils were pelleted in a microcentrifuge tube by spinning down at $12000 \mathrm{rpm}$ for 20 mins. The supernatant was removed and $1 \mathrm{~mL}$ of $\mathrm{D}_{2} \mathrm{O}$ was poured on the pellet. The rinsing cycle was repeated 4-5 times, followed by resuspension of fibrils in $300 \mu \mathrm{D}_{2} \mathrm{O}$ during the last step. Fibrils were then sonicated for $1 \mathrm{~min}$ at 20\% MS72 probe power (Bandelin). FTIR spectra were recorded in a vacuum FTIR spectrophotometer (Bruker). Obtained spectra were processed by subtracting $\mathrm{D}_{2} \mathrm{O}$ reference spectrum and then normalized using GRAMS/AI software (Fisher Scientific).

\section{Data normalization}


184

185

186

187

188

189

190

191

192

193

194

195

196

197

198

199

200

201

202

203

204

205

206

207

208

209

210

211

212

213

214

215

216

Experimental data were normalized using (1) equation:

where $\mathrm{I}_{\text {norm }}$ - normalized ThT fluorescence, I - fluorescence intensity at a certain time point, $\mathrm{I}_{\mathrm{a}}$ and $\mathrm{I}_{\mathrm{b}}-$ minimum and maximum of fluorescence intensity.

\section{Results}

\section{Spontaneous SOD1 aggregation}

The impact of MB was first investigated on the spontaneous aggregation kinetics of SOD1, using a ThT fluorescence assay (Fig. 1A). According to the data, SOD1 aggregation curves have a typical sigmoidal shape and in the control sample (Fig. 1A) the endpoint ThT fluorescence emission value is significantly higher than the background fluorescence, suggesting that long intermolecular $\beta$-sheet structures are being formed. The evidence of fibril formation is shown in an AFM image (Fig. 1B), where several $\mu \mathrm{m}$-long fibrillar structures are visible. MB shows an inhibitory effect on SOD1 aggregation in a concentration-dependent manner, affecting the nucleation phase (as seen by the increase in lag time). Its effect on the quantity of fibrils formed cannot be determined accurately, as MB is known to partially quench the fluorescence of ThT (Ziaunys, Mikalauskaite \& Smirnovas, 2019). Because of the possibility of MB interacting with ThT and affecting both the total fluorescence, as well as aggregation kinetic monitoring, the aggregation reaction was simulateneously tracked by measuring sample absorbance and ThT fluorescence (Fig. S3). Absorbance measurements had a good correlation with the ThT fluorescence assay. Due to this quenching effect, AFM was also employed to confirm aggregate formation and morphology. As the concentration of methylene blue reaches $15 \mu \mathrm{M}$, SOD1 is no longer capable to aggregate into amyloid fibrils within the timescale of the experiment (Fig. 1A and Fig. S3). Interestingly, in high enough concentrations of MB, SOD1 no longer aggregates into amyloid fibrils, but rather forms small oligomers/protofibrils, as seen from an AFM image (Fig. 1C). Furthermore, these small aggregates are not capable of binding several widely used dyes for amyloid detection (Fig. 1-D-F). In the presence of these aggregates, the absorbance spectra of Congo Red dye contains a single peak at $490 \mathrm{~nm}$ and the spectrum is nearly identical to the spectrum of Congo red in just the buffer solution, indicating the presence of an unbound dye, whereas upon fibril binding, a typical peak at $540 \mathrm{~nm}$ appears (Fig. 1D). Nile Red and ThT assays also do not demonstrate an enhanced fluorescence intensity when aggregates are formed in the presence of MB (Fig. 1E, F).

\section{Seeded SOD1 aggregation}


217 Seeded aggregation experiments were conducted to find out whether MB is capable of modifying 218 the SOD1 aggregation pathway when pre-formed fibrils (seeds) are present. In the presence of

219

220

221

222

223

224

225

226

227

228

229

230

231

232

233

234

235

236

237

238

239

240

241

242

243

244

245

246

247

248

249

250

251

252

253

254 seeds, SOD1 aggregates immediately without a lag time, (Fig. 2A-F). At MB concentrations up to $50 \mu \mathrm{M}$, the process of aggregation reaches a plateau within 150 minutes (Fig. 2A-C). At higher MB concentrations, the initial increase is followed by a drop in a ThT fluorescence emission signal (Fig. 2D-F). The morphology of aggregates formed this way is shown in Figure $2 \mathrm{H}$. The most abundant species are small, round-shaped aggregates similar to those formed during the process of spontaneous aggregation (shown in Fig. 1C) and look significantly different from fibrils formed in a control sample (Fig. 2G). Several fibers, visible in Figure 2H, could be traces of the primary fibrillar seed, used to initiate the elongation process. These aggregates, obtained from seeded fibrillization experiments, are also incapable of binding amyloid specific dyes Congo Red, Nile Red and ThT (Fig. 3). Unlike in the spontaneous aggregation experiment, where fibrillization was completely stopped at $15 \mu \mathrm{M} \mathrm{MB}$, seeds were still capable of elongating at $\mathrm{MB}$ concentrations above $15 \mu \mathrm{M}$.

\section{Seeding properties of non-fibrillar SOD1 aggregates formed with MB}

To find out what are the seeding properties of aggregates formed in the presence of $400 \mu \mathrm{M} \mathrm{MB}$, the following aggregation kinetics experiments were carried out. Various fractions (1-10\%) of the aforementioned aggregates were used as seeds to initiate SOD1 monomer fibrillization (Fig. $4 \mathrm{~A})$. In particular, round-shaped aggregates, formed by incubating $200 \mu \mathrm{M}$ SOD 1 with $400 \mu \mathrm{M}$ MB (shown in Fig. 1C), were mixed with monomeric SOD1 in the aggregation favoring buffer and fibrillization kinetics were followed by a ThT assay. As seen in Figure 4A, ThT fluorescence remained at the baseline level even in the presence of $10 \%$ seed and no fibrils were formed (Fig. 4C), whereas in the control samples (SOD1 aggregation initiated by SOD1 fibrils) an immediate rise in ThT signal occurs and fibrils are observed by AFM (Fig. 4B).

\section{MB effect on pre-formed SOD1 fibrils}

In order to test the hypothesis that MB is capable of disassembling SOD1 fibrils, fibers were incubated with $\mathrm{MB}$ in a ratio of 1:2 under optimized aggregation conditions. According to AFM data presented in Figure 5A, SOD1 fibers are present even after 3 days of incubation, indicating that once aggregation occurs, $\mathrm{MB}$ does not disassemble fibrils within the experimental timescale.

To determine alterations in the secondary structure of SOD1 fibrils upon incubation with MB, FTIR spectra of the aggregates were recorded (Fig. 5B, C). SOD1 fibers have a strong signal at $1624 \mathrm{~cm}^{-1}$ (with the main minimum of the second derivative at $1622 \mathrm{~cm}^{-1}$ and a weaker one at $1638 \mathrm{~cm}^{-1}$ ), indicating the dominance of an intermolecular $\beta$-sheet structure. A second derivative minimum at $1669 \mathrm{~cm}^{-1}$, corresponding to a polypeptide chain turn motif is also observed. On the contrary, in the sample of non-aggregated SOD1, a clear single peak at $1633 \mathrm{~cm}^{-1}$ (with the main minimum of the second derivative at $1628 \mathrm{~cm}^{-1}$ and a weaker one at $1646 \mathrm{~cm}^{-1}$ ), is observed, indicating the dominance of a weaker intramolecular $\beta$-sheet structure (Barth, 2007). In the case of preformed fibrils that were incubated with MB for 3 days, the FTIR spectrum main maximum 
255 is at $1632 \mathrm{~cm}^{-1}$, while the second derivative major minimum is at $1624 \mathrm{~cm}^{-1}$, with a weaker one

256 at $1634 \mathrm{~cm}^{-1}$. This implies that the MB-treated fibrils have a secondary structure that is in

257 between the non-aggregated protein and the control fibrils. Interestingly, fibrils incubated with

258 MB were also incapable of binding Congo Red, Nile Red and ThT (Fig. 5D-F), similarly to

259 previously described non-fibrilllar aggregates.

260 As MB affects SOD1 fiber dye-binding properties, which may be related to its interaction with

261 the fibril surface, it is possible that MB-incubated fibrils possess altered seeding properties as

262 well. Therefore, this hypothesis was tested by following seeded aggregation kinetics with a ThT

263 fluorescence assay. According to the data (Fig. 6A-C), MB-incubated SOD1 fibrils elongate at a

264 similar rate as control fibers. These results show that upon incubation with MB, fibril ends

265 remain as active aggregation centers and initiate self-replication of their structure. These ThT-

266 positive aggregates are in the form of elongated fibers (Fig. 6E) and are morphologically similar

267 to fibers formed in a control aggregation mixture (Fig. 6D).

\section{Discussion}

269 When examining the effect of MB on the spontaneous aggregation of SOD1, the data from

270 fibrillization kinetics (Fig. 1A), AFM (Fig. 1B,C) and amyloid dye-binding assays (Fig. 1D-F)

271 suggest that MB not only affects the rate of SOD1 amyloid formation, but also has an impact on

272 the final aggregate structure. The slower association rates, as well as the formation of round-

273 shaped structures that can either be "off-pathway" oligomers or kinetically trapped pre-fibrillar

274 aggregates imply that MB can act as a SOD1 aggregation pathway modulator. Furthermore, the

275 aggregates formed with MB were used to test their seeding propensity (Fig. 4). The baseline

276 level of ThT fluorescence intensity indicates the absence of $\beta$-sheet structures and leads to the

277 conclusion that these structures are incapable of incorporating SOD1 monomers in a templated

278 fashion and therefore are not amyloid-like.

279

280

281

282

283

284

285

286

287

288

289

290

291

292
As MB was capable of inhibiting SOD1 spontaneous fibrillization, its effect was also tested in seeded aggregation conditions (Fig. 2). In this case, two types of effects were observed. Firstly, $15 \mu \mathrm{M}$ of MB no longer had the capacity to fully inhibit fibril growth and much higher concentrations were required to make an effect on SOD1 aggregation kinetics (Fig. 2A-F). This suggests that while MB is capable of modulating nucleation and fibril elongation, it is more potent at inhibiting the former process, rather than the latter one. Secondly, the initial increase in ThT fluorescence intensity was followed by a sudden drop. This could be the result of multiple possible events. The initial hypothesis was that a drop in ThT signal occurs due to a decrease in the fibril mass. However, fibrils incubated with MB demonstrate similar seeding capability as non-incubated counterparts, as indicated by aggregation kinetics experiments (Fig. 6A-C). This is further supported by AFM studies (Fig. 6E), showing the dominance of fibrillar material after pre-formed fibril treatment with MB. According to the second hypothesis, ThT and MB interact with each other either in solution or on the surface of SOD1 aggregates, as a similar phenomenon was shown to occur on the surface of insulin fibrils (Ziaunys, Mikalauskaite \& Smirnovas,

PeerJ reviewing PDF | (2020:05:48576:1:0:NEW 7 Jul 2020) 
293

294

295

296

297

298

299

300

301

302

303

304

305

306

307

308

309

310

311

312

313

314

315

316

317

318

319

320

321

322

323

324

325

326

327

2019). Such interaction may result in ThT fluorescence quenching. However, this scenario does not explain why the fluorescence intensity drop occurs only after a certain time period in a MBconcentration dependent manner (Fig. 2A-F). An alternative explanation would be that MB requires a certain amount of time to change the surface or structure of the formed aggregates, thus impeding their ability to bind amyloid-specific dyes. If this process is significantly slower than fibril elongation, then the drop in intensity would appear after some signal growth is first observed, which is the case in this seeded aggregation experiment. Because of this effect, the kinetic data may not be an accurate representation of the aggregation processes at high MB concentrations.

The hypothesis that MB can change the surface or structure of preformed fibrils was examined by incubating SOD1 fibrils with MB and then comparing their FTIR spectra (Fig. 5B,C), morphology (Fig. 5A), seeding propensity (Fig. 6A-C), as well as dye-binding ability (Fig. 5DF). According to the FTIR spectra, at a first glance it seems like the secondary structure of MBincubated fibrils lies in between the fibrils and monomeric species, as the main maximum in the FTIR spectrum is at $1632 \mathrm{~cm}^{-1}$. However, a second derivative profile of SOD1 MB-treated fiber spectrum (Fig. 5C) is similar to the spectrum of untreated fibrils - the major minimum at 1624 $\mathrm{cm}^{-1}$ and a weaker one at $1634 \mathrm{~cm}^{-1}$ suggest the presence of intermolecular $\beta$-sheets, whereas a minimum at $1669 \mathrm{~cm}^{-1}$ indicates the presence of polypeptide chain turns. This shows that $\mathrm{MB}$ slightly alters SOD1 fibril FTIR spectra, but does not refold their secondary structure. Data from seeding kinetics (Fig. 6A-C) and AFM images (Fig. 6D, E) are in agreement with this conclusion, as their morphology and seeding propensity remain similar to fibrils from the control sample. The major difference is that MB-incubated fibers are incapable of binding amyloidspecific dyes (Fig. 5D-F), similarly to aggregates formed with MB during spontaneous aggregation. Taken together, this leads to the conclusion that once SOD1 fibrils are formed, MB is only capable of interacting with their surface, thus slightly altering their FTIR spectra and dyebinding ability, while having no effect on their morphology and seeding propensity.

\section{Conclusions}

MB has the capacity to not only affect spontaneous SOD1 amyloid aggregation, leading to offpathway species, but it also impedes preformed fibril elongation. Together with the previously reported inhibitory effect of $\mathrm{MB}$ on aggregation of a series of amyloid-forming proteins, our data supports $\mathrm{MB}$ as a potential compound in the search for anti-amyloid drugs.

\section{Acknowledgments}

The authors acknowledge Prof. G. Niaura from the Center of Physical Sciences and Technology for access to FTIR. 
328

329

330

331

332

333

334

335

336

337

338

339

340

341

342

343

344

345

346

347

348

349

350

351

352

353

354

355

356

357

358

359

360

361

362

363

364

365

\section{References}

Abdolvahabi A, Shi Y, Chuprin A, Rasouli S, Shaw BF. 2016. Stochastic Formation of Fibrillar and Amorphous Superoxide Dismutase Oligomers Linked to Amyotrophic Lateral Sclerosis. ACS Chemical Neuroscience 7:799-810. DOI: 10.1021/acschemneuro.6b00048.

Akoury E, Pickhardt M, Gajda M, Biernat J, Mandelkow E, Zweckstetter M. 2013. Mechanistic Basis of Phenothiazine-Driven Inhibition of Tau Aggregation. Angewandte Chemie International Edition 52:3511-3515. DOI: 10.1002/anie.201208290.

Anzai I, Toichi K, Tokuda E, Mukaiyama A, Akiyama S, Furukawa Y. 2016. Screening of Drugs Inhibiting In vitro Oligomerization of $\mathrm{Cu} / \mathrm{Zn}$-Superoxide Dismutase with a Mutation Causing Amyotrophic Lateral Sclerosis. Frontiers in Molecular Biosciences 3:1-11. DOI: 10.3389/fmolb.2016.00040.

Arnesano F, Banci L, Bertini I, Martinelli M, Furukawa Y, O’Halloran T V. 2004. The Unusually Stable Quaternary Structure of Human Cu,Zn-Superoxide Dismutase 1 Is Controlled by Both Metal Occupancy and Disulfide Status. Journal of Biological Chemistry 279:47998-48003. DOI: 10.1074/jbc.M406021200.

Arthur KC, Calvo A, Price TR, Geiger JT, Chiò A, Traynor BJ. 2016. Projected Increase in Amyotrophic Lateral Sclerosis from 2015 to 2040. Nature Communications 7:12408. DOI: $10.1038 /$ ncomms 12408 .

Audet J-N, Soucy G, Julien J-P. 2012. Methylene Blue Administration Fails to Confer Neuroprotection in Two Amyotrophic Lateral Sclerosis Mouse Models. Neuroscience 209:136-143. DOI: 10.1016/j.neuroscience.2011.12.047.

Banci L, Bertini I, Blaževitš O, Calderone V, Cantini F, Mao J, Trapananti A, Vieru M, Amori I, Cozzolino M, Carrì MT. 2012. Interaction of Cisplatin with Human Superoxide Dismutase. Journal of the American Chemical Society 134:7009-7014. DOI: 10.1021/ja211591n.

Barth A. 2007. Infrared Spectroscopy of Proteins. Biochimica et Biophysica Acta (BBA) Bioenergetics 1767:1073-1101. DOI: 10.1016/j.bbabio.2007.06.004.

Bhatia NK, Srivastava A, Katyal N, Jain N, Khan MAI, Kundu B, Deep S. 2015. Curcumin Binds to the Pre-fibrillar Aggregates of $\mathrm{Cu} / \mathrm{Zn}$ Superoxide Dismutase (SOD1) and Alters its Amyloidogenic Pathway Resulting in Reduced Cytotoxicity. Biochimica et Biophysica Acta (BBA) - Proteins and Proteomics 1854:426-436. DOI: 10.1016/j.bbapap.2015.01.014.

Boillée S, Vande Velde C, Cleveland DW. 2006. ALS: A Disease of Motor Neurons and Their Nonneuronal Neighbors. Neuron 52:39-59. DOI: 10.1016/j.neuron.2006.09.018.

Chattopadhyay M, Durazo A, Sohn SH, Strong CD, Gralla EB, Whitelegge JP, Valentine JS. 2008. Initiation and Elongation in Fibrillation of ALS-linked Superoxide Dismutase.

Proceedings of the National Academy of Sciences 105:18663-18668. DOI: 10.1073/pnas.0807058105.

Crowe A, James MJ, Lee VM-Y, Smith AB, Trojanowski JQ, Ballatore C, Brunden KR. 2013. 
401

402

403

Aminothienopyridazines and Methylene Blue Affect Tau Fibrillization via Cysteine Oxidation. Journal of Biological Chemistry 288:11024-11037. DOI:

10.1074/jbc.M112.436006.

Culik RM, Sekhar A, Nagesh J, Deol H, Rumfeldt JAO, Meiering EM, Kay LE. 2018. Effects of Maturation on the Conformational Free-energy Landscape of SOD1. Proceedings of the National Academy of Sciences 115:E2546-E2555. DOI: 10.1073/pnas.1721022115.

Dash RP, Babu RJ, Srinivas NR. 2018. Two Decades-Long Journey from Riluzole to Edaravone: Revisiting the Clinical Pharmacokinetics of the Only Two Amyotrophic Lateral Sclerosis Therapeutics. Clinical Pharmacokinetics 57:1385-1398. DOI: 10.1007/s40262-018-0655-4.

Dibaj P, Zschüntzsch J, Steffens H, Scheffel J, Göricke B, Weishaupt JH, Le Meur K, Kirchhoff F, Hanisch U-K, Schomburg ED, Neusch C. 2012. Influence of Methylene Blue on Microglia-Induced Inflammation and Motor Neuron Degeneration in the SOD1G93A Model for ALS. PLoS ONE 7:e43963. DOI: 10.1371/journal.pone.0043963.

Fang T, Al Khleifat A, Meurgey J-H, Jones A, Leigh PN, Bensimon G, Al-Chalabi A. 2018. Stage at which Riluzole Treatment Prolongs Survival in Patients with Amyotrophic Lateral Sclerosis: a Retrospective Analysis of Data from a Dose-ranging Study. The Lancet Neurology 17:416-422. DOI: 10.1016/S1474-4422(18)30054-1.

Furukawa Y, O’Halloran T V. 2005. Amyotrophic Lateral Sclerosis Mutations Have the Greatest Destabilizing Effect on the Apo- and Reduced Form of SOD1, Leading to Unfolding and Oxidative Aggregation. Journal of Biological Chemistry 280:17266-17274. DOI: 10.1074/jbc.M500482200.

Furukawa Y, Torres AS, O’Halloran T V. 2004. Oxygen-induced Maturation of SOD1: a Key Role for Disulfide Formation by the Copper Chaperone CCS. The EMBO Journal 23:28722881. DOI: $10.1038 /$ sj.emboj.7600276.

Jaiswal MK. 2019. Riluzole and edaravone: A Tale of Two Amyotrophic Lateral Sclerosis Drugs. Medicinal Research Reviews 39:733-748. DOI: 10.1002/med.21528.

Lee S, Kim H-J. 2015. Prion-like Mechanism in Amyotrophic Lateral Sclerosis: are Protein Aggregates the Key? Experimental Neurobiology 24:1. DOI: 10.5607/en.2015.24.1.1.

Liu H-N, Tjostheim S, DaSilva K, Taylor D, Zhao B, Rakhit R, Brown M, Chakrabartty A, McLaurin J, Robertson J. 2012. Targeting of Monomer/Misfolded SOD1 as a Therapeutic Strategy for Amyotrophic Lateral Sclerosis. Journal of Neuroscience 32:8791-8799. DOI: 10.1523/JNEUROSCI.5053-11.2012.

Lougheed R, Turnbull J. 2011. Lack of Effect of Methylene Blue in the SOD1 G93A Mouse Model of Amyotrophic Lateral Sclerosis. PLOS ONE 6:e23141. DOI: 10.1371/journal.pone.0023141.

Maniecka Z, Polymenidou M. 2015. From Nucleation to Widespread Propagation: A Prion-like Concept for ALS. Virus Research 207:94-105. DOI: 10.1016/j.virusres.2014.12.032.

Mattiazzi M, D’Aurelio M, Gajewski CD, Martushova K, Kiaei M, Beal MF, Manfredi G. 2002. 
404

405

406

407

408

409

410

411

412

413

414

415

416

417

418

419

420

421

422

423

424

425

426

427

428

429

430

431

432

433

434

435

436

437

438

439

440

441

Mutated Human SOD1 Causes Dysfunction of Oxidative Phosphorylation in Mitochondria of Transgenic Mice. Journal of Biological Chemistry 277:29626-29633. DOI: 10.1074/jbc.M203065200.

Maurer MS, Schwartz JH, Gundapaneni B, Elliott PM, Merlini G, Waddington-Cruz M, Kristen A V., Grogan M, Witteles R, Damy T, Drachman BM, Shah SJ, Hanna M, Judge DP, Barsdorf AI, Huber P, Patterson TA, Riley S, Schumacher J, Stewart M, Sultan MB, Rapezzi C. 2018. Tafamidis Treatment for Patients with Transthyretin Amyloid Cardiomyopathy. New England Journal of Medicine 379:1007-1016. DOI: 10.1056/NEJMoa1805689.

Miller R, Mitchell J, Lyon M, Moore D. 2002. Riluzole for Amyotrophic Lateral Sclerosis (ALS)/Motor Neuron Disease (MND). Cochrane Database of Systematic Reviews. DOI: 10.1002/14651858.CD001447.

Necula M, Breydo L, Milton S, Kayed R, van der Veer WE, Tone P, Glabe CG. 2007. Methylene Blue Inhibits Amyloid A $\beta$ Oligomerization by Promoting Fibrillization. Biochemistry 46:8850-8860. DOI: 10.1021/bi700411k.

Nowak RJ, Cuny GD, Choi S, Lansbury PT, Ray SS. 2010. Improving Binding Specificity of Pharmacological Chaperones That Target Mutant Superoxide Dismutase-1 Linked to Familial Amyotrophic Lateral Sclerosis Using Computational Methods. Journal of Medicinal Chemistry 53:2709-2718. DOI: 10.1021/jm901062p.

Oz M, Lorke DE, Hasan M, Petroianu GA. 2011. Cellular and Molecular Actions of Methylene Blue in the Nervous System. Medicinal Research Reviews 31:93-117. DOI: 10.1002/med.20177.

Oz M, Lorke DE, Petroianu GA. 2009. Methylene Blue and Alzheimer's Disease. Biochemical Pharmacology 78:927-932. DOI: 10.1016/j.bcp.2009.04.034.

Pansarasa O, Bordoni M, Diamanti L, Sproviero D, Gagliardi S, Cereda C. 2018. SOD1 in Amyotrophic Lateral Sclerosis: "Ambivalent” Behavior Connected to the Disease. International Journal of Molecular Sciences 19:1345. DOI: 10.3390/ijms19051345.

Paré B, Lehmann M, Beaudin M, Nordström U, Saikali S, Julien J-P, Gilthorpe JD, Marklund SL, Cashman NR, Andersen PM, Forsberg K, Dupré N, Gould P, Brännström T, Gros-Louis F. 2018. Misfolded SOD1 Pathology in Sporadic Amyotrophic Lateral Sclerosis. Scientific Reports 8:14223. DOI: 10.1038/s41598-018-31773-z.

Peters OM, Brown Jr RH. 2015. Neurobiology of Brain Disorders. Elsevier Science Publishing 1:262-279.

Pickles S, Semmler S, Broom HR, Destroismaisons L, Legroux L, Arbour N, Meiering E, Cashman NR, Vande Velde C. 2016. ALS-linked Misfolded SOD1 Species have Divergent Impacts on Mitochondria. Acta Neuropathologica Communications 4:43. DOI: 10.1186/s40478-016-0313-8.

Rakhit R, Chakrabartty A. 2006. Structure, Folding, and Misfolding of $\mathrm{Cu}, \mathrm{Zn}$ Superoxide 
Dismutase in Amyotrophic Lateral Sclerosis. Biochimica et Biophysica Acta (BBA) Molecular Basis of Disease 1762:1025-1037. DOI: 10.1016/j.bbadis.2006.05.004.

Ray SS, Nowak RJ, Brown RH, Lansbury PT. 2005. Small-molecule-mediated Stabilization of Familial Amyotrophic Lateral Sclerosis-linked Superoxide Dismutase Mutants Against Unfolding and Aggregation. Proceedings of the National Academy of Sciences 102:36393644. DOI: $10.1073 /$ pnas.0408277102.

Renton AE, Chiò A, Traynor BJ. 2014. State of Play in Amyotrophic Lateral Sclerosis Genetics. Nature Neuroscience 17:17-23. DOI: 10.1038/nn.3584.

Rosen DR, Siddique T, Patterson D, Figlewicz DA, Sapp P, Hentati A, Donaldson D, Goto J, O’Regan JP, Deng H-X, Rahmani Z, Krizus A, McKenna-Yasek D, Cayabyab A, Gaston SM, Berger R, Tanzi RE, Halperin JJ, Herzfeldt B, Van den Bergh R, Hung W-Y, Bird T, Deng G, Mulder DW, Smyth C, Laing NG, Soriano E, Pericak-Vance MA, Haines J, Rouleau GA, Gusella JS, Horvitz HR, Brown RH. 1993. Mutations in Cu/Zn Superoxide Dismutase Gene are Associated with Familial Amyotrophic Lateral Sclerosis. Nature 362:59-62. DOI: 10.1038/362059a0.

Rothstein JD. 2017. Edaravone: A New Drug Approved for ALS. Cell 171:725. DOI: 10.1016/j.cell.2017.10.011.

Rotunno MS, Bosco D a. 2013. An Emerging Role for Misfolded Wild-type SOD1 in Sporadic ALS Pathogenesis. Frontiers in Cellular Neuroscience 7:253. DOI: 10.3389/fncel.2013.00253.

Saha B, Chowdhury S, Sanyal D, Chattopadhyay K, Suresh Kumar G. 2018. Comparative Study of Toluidine Blue $\mathrm{O}$ and Methylene Blue Binding to Lysozyme and Their Inhibitory Effects on Protein Aggregation. ACS Omega 3:2588-2601. DOI: 10.1021/acsomega.7b01991.

Sheng Y, Chattopadhyay M, Whitelegge J, Selverstone Valentine J. 2013. SOD1 Aggregation and ALS: Role of Metallation States and Disulfide Status. Current Topics in Medicinal Chemistry 12:2560-2572. DOI: 10.2174/1568026611212220010.

Smith BN, Topp SD, Fallini C, Shibata H, Chen H-J, Troakes C, King A, Ticozzi N, Kenna KP, Soragia-Gkazi A, Miller JW, Sato A, Dias DM, Jeon M, Vance C, Wong CH, de Majo M, Kattuah W, Mitchell JC, Scotter EL, Parkin NW, Sapp PC, Nolan M, Nestor PJ, Simpson M, Weale M, Lek M, Baas F, Vianney de Jong JM, ten Asbroek ALMA, Redondo AG, Esteban-Pérez J, Tiloca C, Verde F, Duga S, Leigh N, Pall H, Morrison KE, Al-Chalabi A, Shaw PJ, Kirby J, Turner MR, Talbot K, Hardiman O, Glass JD, De Belleroche J, Maki M, Moss SE, Miller C, Gellera C, Ratti A, Al-Sarraj S, Brown RH, Silani V, Landers JE, Shaw CE. 2017. Mutations in the Vesicular Trafficking Protein Annexin A11 are Associated with Amyotrophic Lateral Sclerosis. Science Translational Medicine 9:eaad9157. DOI: 10.1126/scitranslmed.aad9157.

Sontag EM, Lotz GP, Agrawal N, Tran A, Aron R, Yang G, Necula M, Lau A, Finkbeiner S, Glabe C, Marsh JL, Muchowski PJ, Thompson LM. 2012. Methylene Blue Modulates Huntingtin Aggregation Intermediates and Is Protective in Huntington's Disease Models. Journal of Neuroscience 32:11109-11119. DOI: 10.1523/JNEUROSCI.0895-12.2012. 
482

483

484

485

486

487

488

489

490

491

492

493

494

495

496

497

498

499

500

501

502

503

504

505

506

507

508

509

510

511

512

513

514

515

Srinivasan E, Rajasekaran R. 2017. Probing the Inhibitory Activity of Epigallocatechin-gallate on Toxic Aggregates of Mutant (L84F) SOD1 Protein through Geometry Based Sampling and Steered Molecular Dynamics. Journal of Molecular Graphics and Modelling 74:288295. DOI: 10.1016/j.jmgm.2017.04.019.

Studier FW. 2005. Protein Production by Auto-induction in High-density Shaking Cultures. Protein Expression and Purification 41:207-234. DOI: 10.1016/j.pep.2005.01.016.

Taylor JP, Brown RH, Cleveland DW. 2016. Decoding ALS: from Genes to Mechanism. Nature 539:197-206. DOI: 10.1038/nature20413.

Vaccaro A, Patten SA, Aggad D, Julien C, Maios C, Kabashi E, Drapeau P, Parker JA. 2013. Pharmacological Reduction of ER Stress Protects against TDP-43 Neuronal Toxicity In vivo. Neurobiology of Disease 55:64-75. DOI: 10.1016/j.nbd.2013.03.015.

Vaccaro A, Patten SA, Ciura S, Maios C, Therrien M, Drapeau P, Kabashi E, Parker JA. 2012. Methylene Blue Protects against TDP-43 and FUS Neuronal Toxicity in C. elegans and D. rerio. PLoS ONE 7:e42117. DOI: 10.1371/journal.pone.0042117.

Wischik CM, Edwards PC, Lai RY, Roth M, Harrington CR. 1996. Selective Inhibition of Alzheimer Disease-like Tau Aggregation by Phenothiazines. Proceedings of the National Academy of Sciences 93:11213-11218. DOI: 10.1073/pnas.93.20.11213.

Wright GSA, Antonyuk S V, Kershaw NM, Strange RW, Samar Hasnain S. 2013. Ligand Binding and Aggregation of Pathogenic SOD1. Nature Communications 4:1758. DOI: 10.1038/ncomms2750.

Yamashita M, Nonaka T, Arai T, Kametani F, Buchman VL, Ninkina N, Bachurin SO, Akiyama H, Goedert M, Hasegawa M. 2009. Methylene Blue and Dimebon Inhibit Aggregation of TDP-43 in Cellular Models. FEBS Letters 583:2419-2424. DOI: 10.1016/j.febslet.2009.06.042.

Zarei S, Carr K, Reiley L, Diaz K, Guerra O, Altamirano P, Pagani W, Lodin D, Orozco G, Chinea A. 2015. A Comprehensive Review of Amyotrophic Lateral Sclerosis. Surgical Neurology International 6:171. DOI: 10.4103/2152-7806.169561.

Zhuang X, Zhao B, Liu S, Song F, Cui F, Liu Z, Li Y. 2016. Noncovalent Interactions between Superoxide Dismutase and Flavonoids Studied by Native Mass Spectrometry Combined with Molecular Simulations. Analytical Chemistry 88:11720-11726. DOI: 10.1021/acs.analchem.6b03359.

Ziaunys M, Mikalauskaite K, Smirnovas V. 2019. Amyloidophilic Molecule Interactions on the Surface of Insulin Fibrils : Cooperative Binding and Fluorescence Quenching. Scientific Reports:1-10. DOI: 10.1038/s41598-019-56788-y. 


\section{Figure 1}

Spontaneous SOD1 aggregation.

SOD1 aggregation kinetics followed by ThT (A); SOD1 aggregated in $10 \mathrm{mM}$ potassium phosphate buffer with $0.5 \mathrm{M} \mathrm{GuHCl}$ and $5 \mathrm{mM}$ DTT with 0-15 $\mu \mathrm{M} \mathrm{MB}, \mathrm{pH}$ 7.4. AFM images of aggregates formed without (B) and with (C) $15 \mu \mathrm{M}$ MB. CR absorbance spectrum (D), as well as NR (E) and ThT (F) fluorescence spectra of aggregates formed with MB.
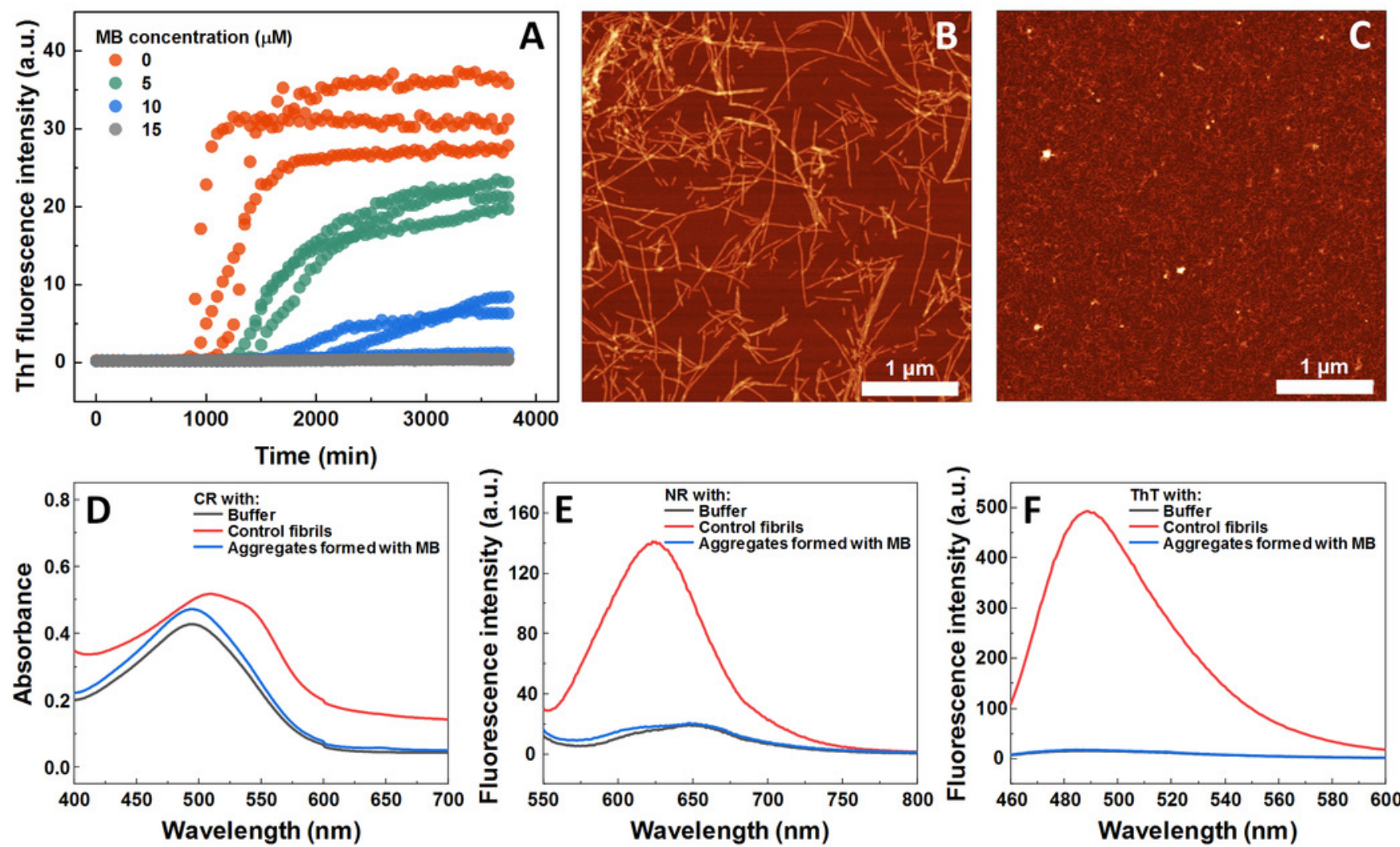
Figure 2

Seeded SOD1 aggregation.

Aggregation kinetics followed by ThT, when 0 (A), 25 (B), 50 (C), 100 (D), 200 (E) and $400 \mu \mathrm{M}$ (F) of MB is present in the sample; SOD1 aggregated with $5 \%$ (of total protein in the sample) seed in $10 \mathrm{mM}$ potassium phosphate buffer with $0.5 \mathrm{M} \mathrm{GuHCl}$ and $5 \mathrm{mM}$ DTT with 0-400 $\mu \mathrm{M}$ methylene blue, $\mathrm{pH}$ 7.4. Three repeats for each condition are represented by different colors. AFM images of aggregates formed without $(\mathrm{G})$ and with $(\mathrm{H}) 400 \mu \mathrm{M}$ methylene blue. 

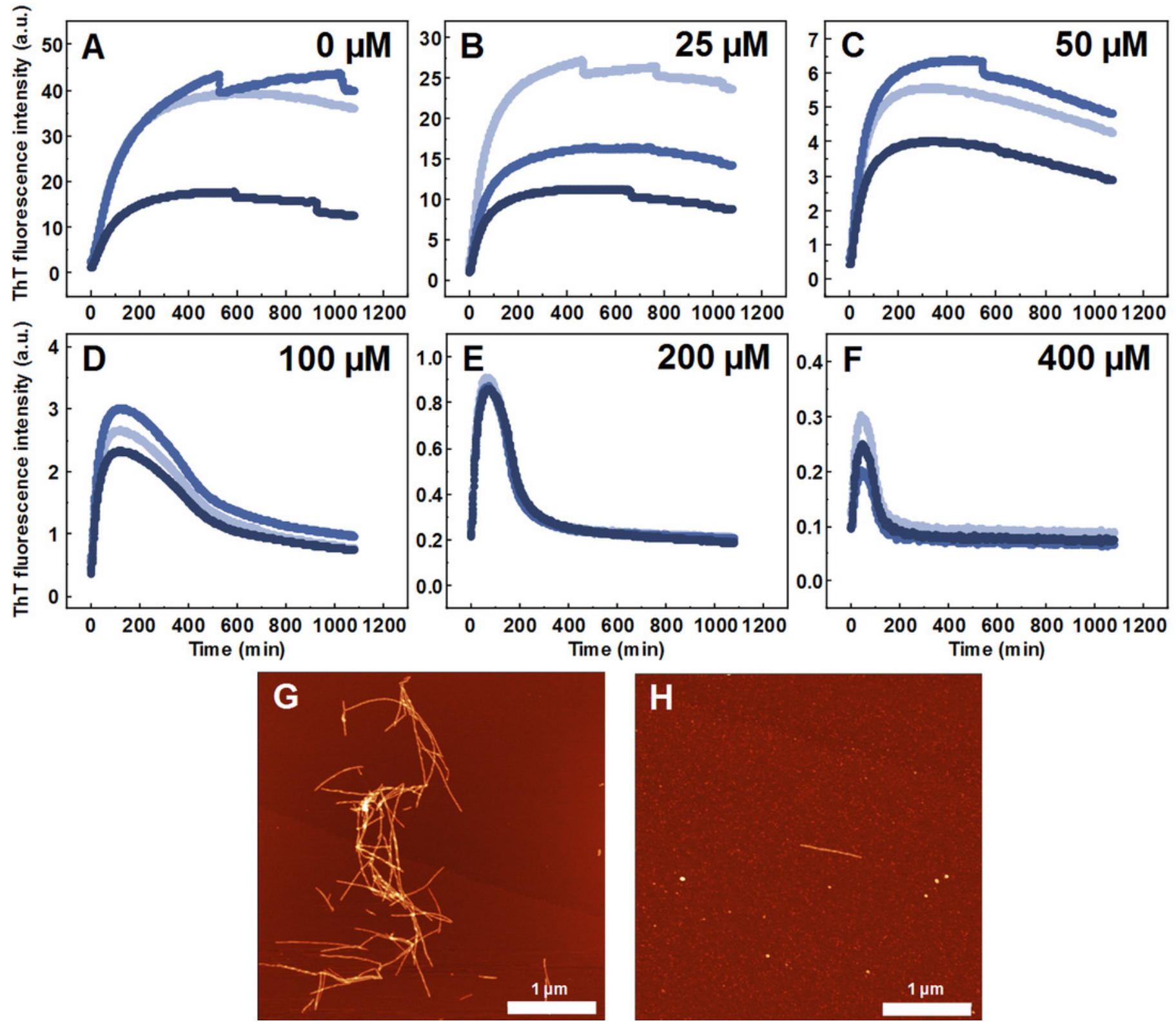
Figure 3

Dye binding assay of aggregates formed by reseeding SOD1 fibrils in the presence of MB.

CR (A) absorbance spectrum, as well as NR (B) and ThT (C) fluorescence spectra.
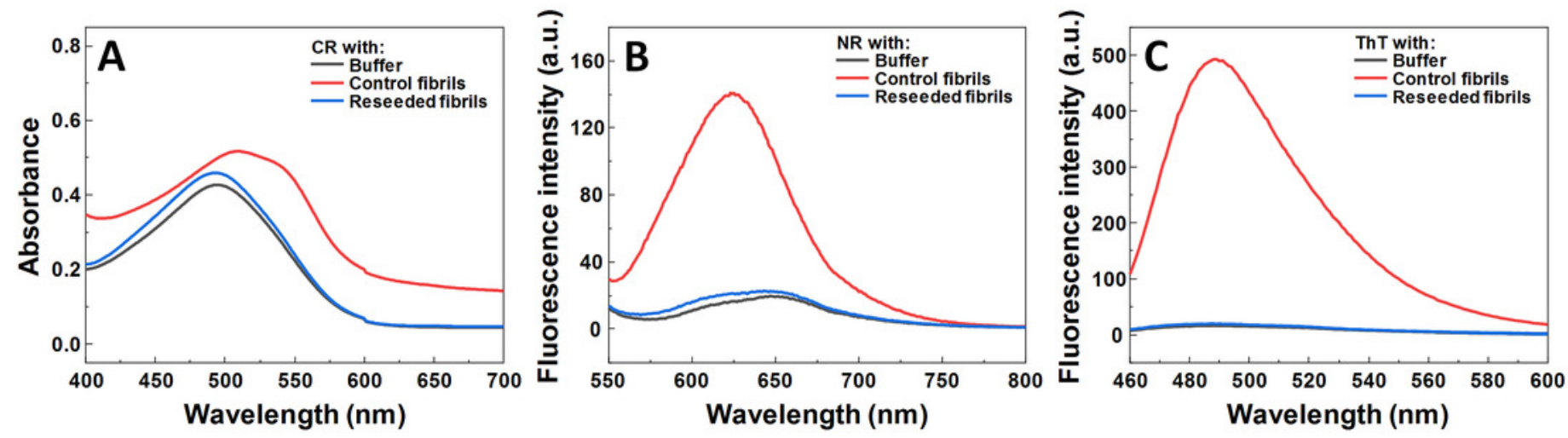


\section{Figure 4}

Seeded SOD1 aggregation, where aggregates formed with MB were used as seed.

Aggregation kinetics followed by ThT (A); SOD1 aggregated with 1-10\% seed in $10 \mathrm{mM}$ potassium phosphate buffer with $0.5 \mathrm{M} \mathrm{GuHCl}$ and $5 \mathrm{mM} \mathrm{DTT}, \mathrm{pH}$ 7.4. The seed was prepared by incubating SOD1 with $400 \mu \mathrm{M}$ methylene blue. Control samples contained an equivalent concentration of MB as the samples with the MB-formed aggregates. AFM images from the control experiment (B) and when fibrils formed with MB were used as seed (C).
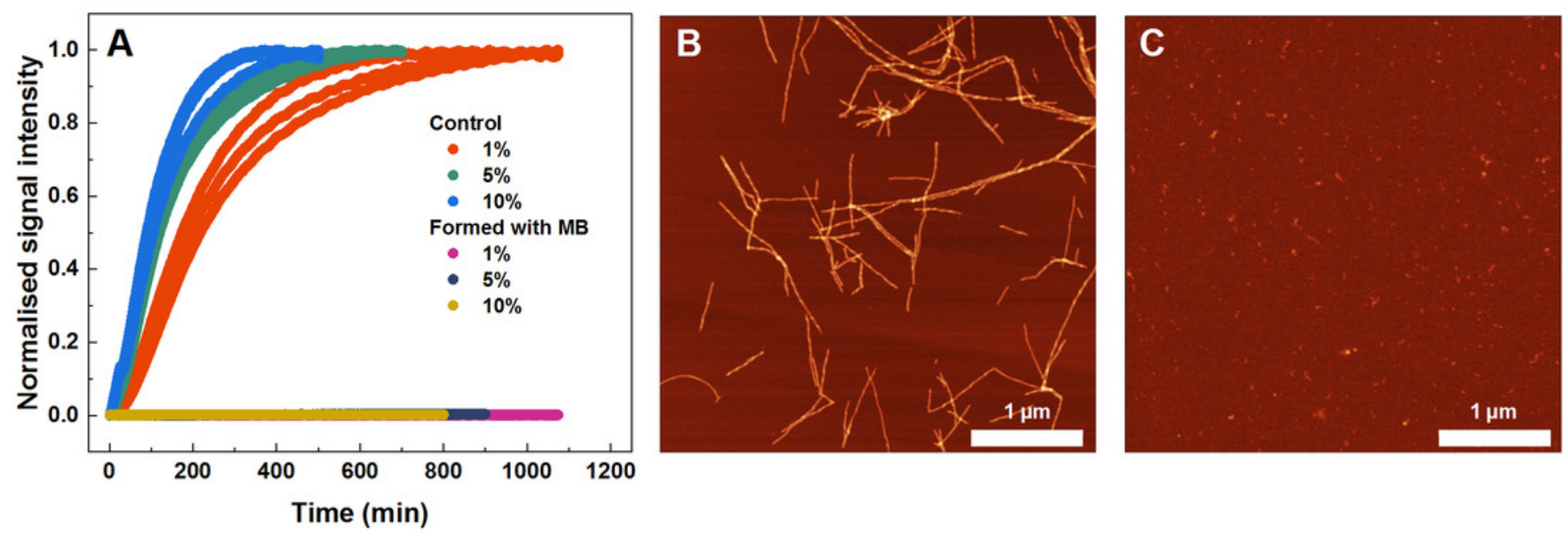


\section{Figure 5}

Effect of MB on SOD1 fibril morphology and FTIR spectra.

AFM image of $200 \mu \mathrm{M}$ preformed SOD1 fibrils incubated with $400 \mu \mathrm{M}$ methylene blue for 3 days (A), FTIR (B) and second derivative (C) spectra of SOD1 fibrils and monomer. CR absorbance spectrum (D), as well as NR (E) and ThT (F) fluorescence spectra of aggregates formed with MB.
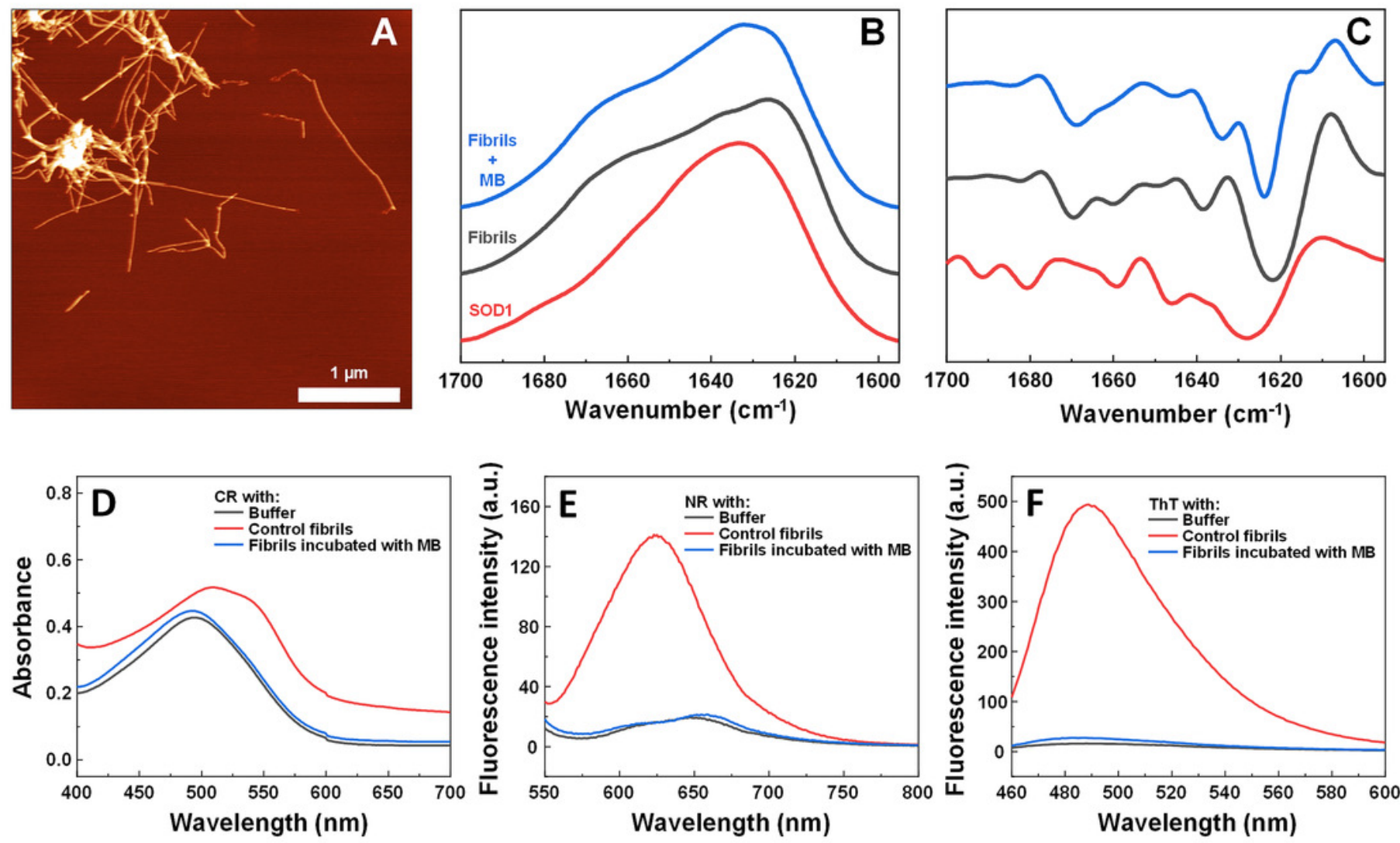


\section{Figure 6}

Seeded SOD1 aggregation, when fibrils incubated with MB were used as seed.

Aggregation kinetics followed by ThT ; SOD1 aggregated with 1\% (A), 5\% (B) and 10\% (C) seed in $10 \mathrm{mM}$ potassium phosphate buffer with $0.5 \mathrm{M} \mathrm{GuHCl}$ and $5 \mathrm{mM}$ DTT, pH 7.4. Control samples contained an equivalent concentration of MB as the samples with MB-treated fibrils. AFM images of aggregates formed with control (D) and MB-treated fibrils (E).
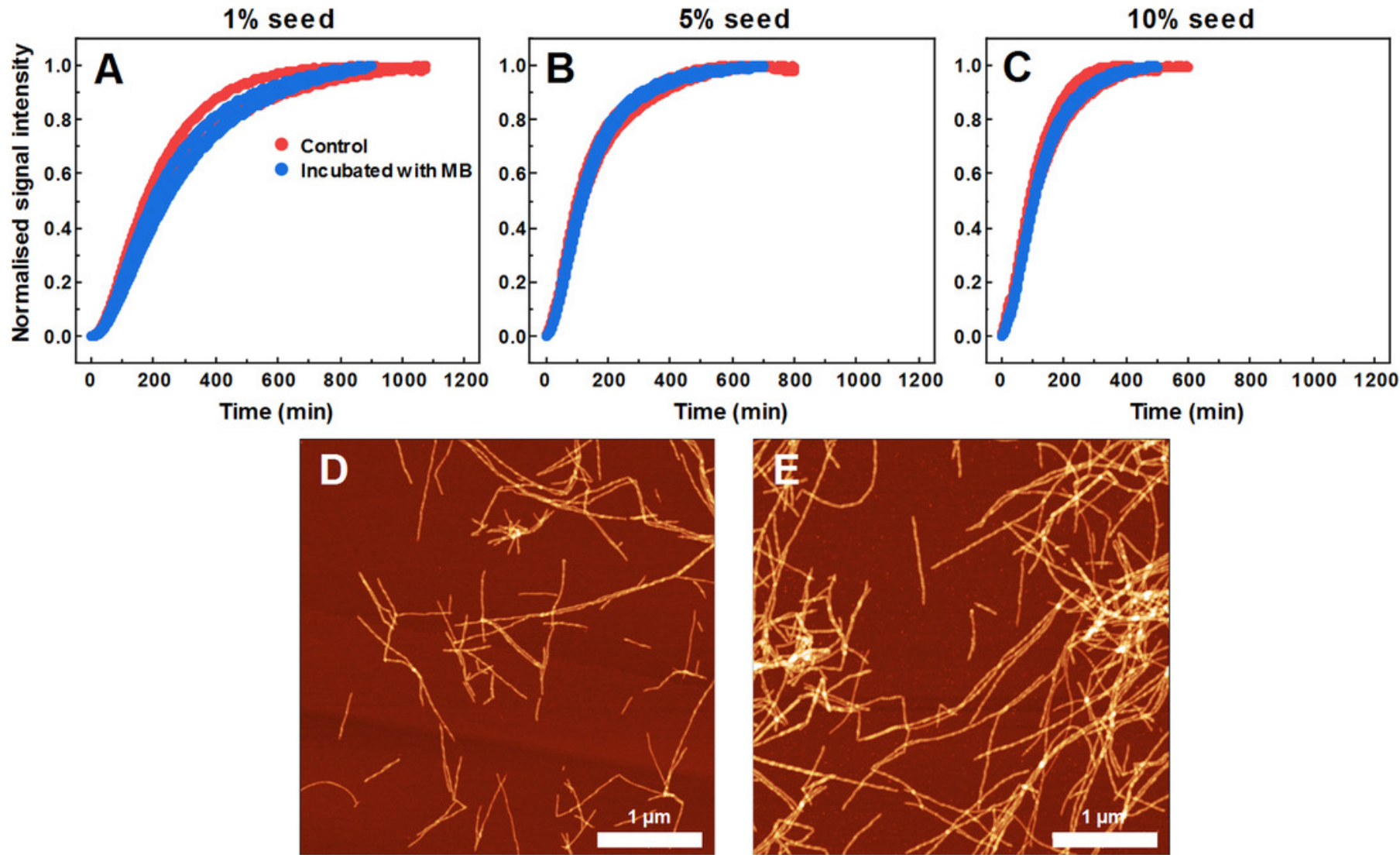